\title{
DEVELOPMENT OF A MATHEMATICAL MODEL FOR AUTOMATING THE SELECTION OF EFFECTIVE TECHNOLOGICAL ZONES FOR THE PLACEMENT OF PTM COMPONENTS WITH VISCOELASTIC LINKS IN DYNAMIC CONTROL
}

\author{
Alisher Mamadjanov ${ }^{1}$, Zayniddin Mukhiddinov ${ }^{1}$, Sarvar Nabiyev ${ }^{1}$ \\ ${ }^{1}$ Tashkent State Technical University \\ Email:fff8uma@mail.ru
}

\begin{abstract}
This work is devoted to the development of a mathematical model that allows automating the processes of selecting effective technological zones for placing components of production and technological modules (PTM) with viscoelastic links in dynamic control. The main principles of creating and organizing automated production and technological systems are: the principle of combining high productivity and versatility; the principle of modularity; the principle of hierarchical subordination and the principle of ensuring a high level of compatibility.
\end{abstract}

Keywords: Industrial robot, Storage system, Component, Automation, Dynamic control, Functional-parametric compatibility.

\section{Introduction}

Automated production and technological systems (PTS) play a particularly important role in the development of machine-building production. One of the main principles of creating and organizing automated PTS is the principle of ensuring a high level of compatibility of their components.

In this regard, this paper considers the issues of automation of the processes of selecting effective technological zones for placing components PTM with viscoelastic links in dynamic control with simultaneous fulfillment of the requirements of functional-parametric compatibility (FPS) of interacting components of the PTS, in particular, PTM of mechanical processing of machine-building production.

Many works are devoted to the issues of determining and ensuring the FPC of PTS components, including PTM $[1,2,3,4]$.

In [3], a method for determining and ensuring the FPC of PTM components was developed, based on an analytical approach for the mechanical model of the manipulator, i.e., under the assumption that all the links of the manipulator are considered rigid. It is known that recently composite and other materials with viscoelasticity properties and providing high reliability of their functioning have been widely used in the designs of manipulators.

\section{Methods}

Let's consider the kinematic scheme of an industrial robot (IR), which is a rectilinear rods $P_{1}, P_{2}, \cdots, P_{n}{ }^{\prime}$ connected by cylindrical hinges $L_{1}, L_{2}, \cdots, L_{n+1} \cdot$

At the same time $L_{1}$ it is attached to a fixed base, i.e. to a fixed coordinate system $O_{0} x_{0} y_{0} z_{0}$, and the hinge, $L_{n+1}$ connects the working body (gripping device) IR.

To describe the motion of the system, the generalized coordinates are selected $q^{(1)}, q^{(2)}, \cdots, q^{(n+1)}$.

Consider the functions $f_{1}, f_{2}, \cdots, f_{n}$ and the matrix ${ }_{F=\left(F_{i j}\right)}$, for which there are:

$$
q_{i}=f_{i}\left(\alpha_{i}\right)+x_{i}, \quad F_{i j}=\frac{\partial f_{i}\left(\alpha_{j}\right)}{\partial \alpha_{j}}
$$

Then the dynamics of the elastic manipulator can be set by the following relations: 


$$
\left.\begin{array}{c}
\frac{d}{d t}[A(q) \cdot \dot{q}]-(A(q) \cdot \dot{q}, \dot{q})=Q(q, \dot{q}, t)-C(\alpha) x \\
M=-F^{\prime}(\alpha) C(\alpha) x, \quad(x=q-f(\alpha)), \\
M=F^{\prime}(\alpha)\left\{\frac{d}{d t}[A(q) \cdot \dot{q}]-\frac{1}{2} \frac{\partial}{\partial q}[A(q) \cdot \dot{q}, \dot{q}]-Q(q, \dot{q}, t)\right\} \\
C(\alpha)=\varepsilon^{-2} K(\alpha), x=\varepsilon^{2} X, \varepsilon<<1
\end{array}\right\}
$$

where $A, C$ - symmetric positive-definite matrices;

$Q(q, \dot{q}, t)=\left(Q_{1}, Q_{2}, \cdots, Q_{n}\right)$ - vector of external generalized forces;

$K, X$ - a matrix and a vector with elements of the order of one, respectively [5].

Now let's consider the problem of determining and ensuring functional - parametric compatibility, when the control is set by kinematic parameters, i.e. the laws of angle change are set $\alpha=\alpha^{0}(t)$ and movements $\xi=\xi^{0}(t)$ in the joints and links, respectively.

Then, for PTM components with viscoelastic links, there is:

$$
\begin{aligned}
& \frac{d}{d t}\left[A\left(q^{0}+\varepsilon^{2} X\right) \cdot\left(\dot{q}^{0}+\varepsilon^{2} X\right)\right]-\frac{1}{2} \frac{\partial}{\partial q}\left[A\left(q^{0}+\varepsilon^{2} X\right)\left(\dot{q}^{0}+\varepsilon^{2} X\right)\right]\left(q^{0}+\right. \\
& \left.+\varepsilon^{2} X\right)=Q\left(\left(q^{0}+\varepsilon^{2} X, t\right)-K\left(\alpha^{0}\right) X=\alpha=\alpha^{0}(t), q^{0}(t)=f\left(\alpha^{0}(t)\right)\right.
\end{aligned}
$$

here $q^{0}(t)$ - the law of motion of the gripper device in the case of a mechanical model. The solution (3) can be represented as [5]

$$
X(t)=R_{1}(t)+R_{2}(\tau), \quad \tau=t / \varepsilon
$$

where are the terms $R_{1}(t)$ describes the offsets that occur during manipulation operations, and $R_{2}(\tau)$ describes elastic vibrations having a time order $\varepsilon^{-1}$.

Let's define the FPC function $i$-th the link of the IR with the MTE in the following form

$$
\begin{aligned}
& \Delta^{(i)}(t, \tau)=\Delta_{12}^{(i)}(t, \tau) \Delta_{12}^{(i)}(t, \tau) \Delta_{12}^{(i)}(t, \tau), \\
& 0<t<T_{0}, \tau=t / \varepsilon
\end{aligned}
$$

Where:

$$
\begin{aligned}
\Delta_{12}^{(i)}(t, \tau)= & \partial \boldsymbol{W}(x, y, z) / \partial x \cdot \partial \boldsymbol{P}(x, y, z) / \partial y- \\
& -\partial \boldsymbol{W}(x, y, z) / \partial y \cdot \partial \boldsymbol{P}(x, y, z) /\left.\partial x\right|_{R^{(i)}=R_{01}^{(i)}(t)+R_{02}^{(i)}(\tau)} \\
\Delta_{23}^{(i)}(t, \tau)= & \partial \boldsymbol{W}(x, y, z) / \partial y \cdot \partial \boldsymbol{P}(x, y, z) / \partial z- \\
& -\partial \boldsymbol{W}(x, y, z) / \partial z \cdot \partial \boldsymbol{P}(x, y, z) / \partial y \mid \\
& \\
\Delta_{31}^{(i)}(t, \tau)= & \partial \boldsymbol{W}(x, y, z) / \partial z \cdot \partial \boldsymbol{P}(x, y, z) / \partial x- \\
& -\partial \boldsymbol{W}(x, y, z) / \partial x \cdot \partial \boldsymbol{P}(x, y, z) /\left.\partial z\right|_{01} ^{(i)}=R_{01}^{(i)}(t)+R_{02}^{(i)}(\tau)
\end{aligned}
$$

where $R_{01}^{(i)}(t)=\left(x_{01}^{(i)}(t), y_{01}^{(i)}(t), z_{01}^{(i)}(t)\right)$ describes the displacements that occur during manipulation operations of the $i$-th link relative to the coordinate system $O_{0} x_{0} y_{0} z_{0}$, connected to the fixed base of the IR, and $R_{02}^{(i)}(\tau)=\left(x_{02}^{(i)}(\tau), y_{02}^{(i)}(\tau), z_{02}^{(i)}(\tau)\right) \quad(\tau=t / \varepsilon)$ describes elastic vibrations of the $\mathrm{i}$-th link relative to the coordinate system $O_{0} x_{0} y_{0} z_{0}$, connected to the fixed base of the IR.

$R^{(i)}(t, \tau)=\left(x^{(i)}(t, \tau), y^{(i)}(t, \tau), z^{(i)}(t, \tau)\right) \quad-\quad$ a vector that determines the spatial position of some point of the $i$-th link relative to the coordinate system $O_{0} x_{0} y_{0} z_{0}$, connected to the fixed base of the IR. 
Thus, we have the FPC function of the $i$-th IR link with viscoelastic links with MTE $\Delta^{(i)}(t, \tau)$.

The definition of FPC IR with MTE is implemented by checking the condition $\Delta^{(i)}(t, \tau) \neq 0$ by $0<t<T_{0}, \tau=t / \varepsilon$.

It should be noted that the function $\Delta^{(i)}(t, \tau)$ it is a generalization of the function obtained in [3, 7]. Indeed, in the special case when the mechanical model of the IR is considered, its links are considered rigid and, obviously, that

$$
R_{02}^{(i)}(\tau)=0 \text {, т.e. }
$$

$\Delta^{(i)}(t, \tau)=\Delta^{(i)}(t)$

\section{Results and Discussions}

The developed model summarizes the results obtained in $[3,7]$, and the principle of developing algorithms for automating the processes of determining and ensuring functional-parametric compatibility of viscoelastic components of PTM remains the same. The difference is that at the stage of calculating the value of the FPC function $\Delta^{(i)}(t, \tau)$ the terms are added

$$
R_{02}^{(i)}(\tau)=\left(x_{02}^{(i)}(\tau), y_{02}^{(i)}(\tau), z_{02}^{(i)}(\tau)\right) \quad(\tau=t / \varepsilon) .
$$

It is known that the dynamic control of the IR is carried out by finding the laws of motion of its executive bodies for given laws of change of the moments of the drives $M=M_{0}(\alpha, t)$. At the same time, it can be assumed that this case includes both software control $M=M_{0}(t)$; and the so-called feedback control $M=M_{0}(\alpha, t)$, where $\alpha=\left(\alpha_{1}, \alpha_{2}, \cdots, \alpha_{n}\right)$ - the vector of angular values in the joints of the links of the IR.

Thus, it is required to find the movement of the executive bodies of the loaded IR, taking into account the above-mentioned requirements of the FPC, i.e. $q(t)$ and $q(t)=\left(q_{1}(t), q_{2}(t), \cdots, q_{n}(t)\right)$ - generalized coordinates of the spatial position of the cargo.

$$
\text { Let } f=\left(f_{1}, f_{2}, \cdots, f_{n}\right) \text { and } g=\left(g_{1}, g_{2}, \cdots, g_{n}\right)^{-}
$$

vector functions and $F=\left\{F_{i j}\right\}^{\text {и }} G=\left\{G_{i j}\right\}^{\text {- matrices }}$ for which there are

$$
\begin{gathered}
q=f(\alpha), \quad\left(q_{i}=f_{i}(\alpha)\right), \\
F=\left\{F_{i j}\right\}, \quad F_{i j}(\alpha)=\partial f_{i}(\alpha) / \partial \alpha_{j}, \\
g(q)=g\left(f(\alpha)=\alpha, \quad g=f^{-1}(\alpha)\right), \\
G=\left\{G_{i j}\right\}=\left\{\partial g_{i}(\alpha) / \partial \alpha_{j}\right\}, G=F^{-1}
\end{gathered}
$$

Now let's consider the kinematic scheme of the IR with elastic links, representing rectilinear rods $P_{1}, P_{2}, \cdots, P_{n}$, connected by cylindrical hinges $L_{1}, L_{2}, \cdots, L_{n+1}$. At the same time $L_{1}$ it is attached to a fixed base, i.e. to a fixed coordinate system $O_{0} x_{0} y_{0} z_{0}$, connected to the base of the IR, and the hinge $L_{n+1}$ connects the working body (gripper device) IR [8].

We will accept

$$
q=f(\alpha)+s, \quad s=\left(s_{1}, s_{2}, \cdots, s_{n}\right),
$$

where $S_{i}$-values of small elastic displacements of the load relative to the $i$-th coordinate system $O_{i} x_{i} y_{i} z_{i}$, related to the $i$-th link of the IR [9].

The Lagrange equation is presented in the following form

$$
M_{0}(\alpha, t)=-\varepsilon^{2} F^{\prime}(\alpha(t)) \Phi(\alpha(t)) s,
$$

where $s=\varepsilon^{2} S$,

$\Phi(\alpha(t)), X-$ a matrix and a vector of the order of one.

From here we get:

$$
\begin{gathered}
s=-\varepsilon^{2}\left[F^{\prime}(\alpha(t)) \Phi(\alpha(t))\right]^{-1} M_{0}(\alpha, t), q=f(\alpha)+s \\
\alpha(t)=g(q(t))+G(q(t)) \Phi^{-1}\left(g(q(t))+G^{\prime}(q(t))\right) M_{0}(g(q(t)), t)
\end{gathered}
$$

Taking into account (6)-(9), the equation of motion of the executive body of the IR with elastic links can be represented as:

$$
L=Q(q, \dot{q}, t)+G^{\prime}(q(t)) M_{0}(g(q(t)), t)++\varepsilon^{2} \Theta(q(t), t) \Phi^{-1}\left(g(q(t)) G^{\prime}(q(t))\right) M_{0}(g(q(t)), t)
$$

where:

$$
\Theta(q(t), t)=\left\{\partial G_{i j}^{\prime}(q(t)) M_{\mathrm{O}}\left(g(q(t), t) / \partial q_{j}(t)\right\}, i, j=\overline{1, n}\right.
$$

Taking into account:

$q=f(\alpha), \quad G=F^{-1}(\alpha), M_{0}=M_{0}(\alpha, t)$

equation (10) you can write it in the following form:

$$
\begin{aligned}
& L=Q(\alpha, \dot{\alpha}, t)+\left(F^{-1}\right)^{\prime}(f(\alpha)) M_{0}(\alpha, t)+ \\
& \left.+\varepsilon^{2} \Theta(f(\alpha), t) \Phi^{-1}(\alpha) G^{\prime}(f(\alpha))\right) M_{0}(\alpha, t)
\end{aligned}
$$


Equation (11) is solved with respect to $\alpha=\left(\alpha_{1}, \alpha_{2}, \cdots, \alpha_{n}\right)$, which are equivalent for both mechanical and elastic models due to the fact that the angles in the hinges are the same for both models.

Thus $\alpha(t)=\Psi\left(\breve{Q}, M_{0}, \Theta, \Phi^{-1}, G^{\prime}, f, \varepsilon^{2}, t\right), \Psi$ - the vector is a function defined taking into account the types of displacements in the links of the IR.

The method of constructing coordinate systems associated with the working links of the IR is given in [9].

According to the results of this work, the transition from one coordinate system to another without providing the FPC of the $i$-th link of the IR with the main technological unit (MTE) is given by the following formula

$$
R_{i-1} \alpha(t)=T_{i}(\alpha(t)) R_{i}
$$

where $R_{i}$ - radius is a vector that determines the spatial position of some point of the $i$-th link relative to the coordinate system associated with this link;
$T_{i}(\alpha(t))$ - the transition matrix from the coordinate system associated with the $i$-th link to the coordinate system associated with the $(i-1)$ th.

Then it is possible to determine the spatial position of some point of the $i$-th link $P_{i}$ relative to the coordinate system associated with the fixed base of the IR $O_{0} x_{0} y_{0} z_{0}$ as follows

$$
R_{i}^{0}(t)=\Gamma_{i}\left(\alpha_{1}(t), \alpha_{2}(t), \cdots, \alpha_{i}(t)\right) R_{i}(t),
$$

where:

$$
\begin{aligned}
& \Gamma_{i}\left(\alpha_{1}(t), \alpha_{2}(t), \cdots, \alpha_{i}(t)\right)=T_{1}\left(\alpha_{1}\right) \cdot T_{2}\left(\alpha_{2}\right) \cdots \cdots T_{i}\left(\alpha_{i}\right), \\
& R_{i}^{0}(t)=\left(x_{i}^{0}(t), y_{i}^{0}(t), z_{i}^{0}(t)\right), \quad R_{i}(t)=\left(x_{i}(t), y_{i}(t), z_{i}(t)\right) .
\end{aligned}
$$

We define the FPC function of the $i$-th link of the IR with the MTE in the following form

$$
\Delta^{(i)}(t, \tau)=\Delta_{12}^{(i)}(t, \tau) \Delta_{12}^{(i)}(t, \tau) \Delta_{12}^{(i)}(t, \tau), 0<t<T_{0}, \tau=t / \varepsilon
$$

where:

$$
\begin{aligned}
\Delta_{12}^{(i)}(t, \tau)= & \partial \boldsymbol{W}(x, y, z) / \partial x \cdot \partial \boldsymbol{P}(x, y, z) / \partial y- \\
& -\partial \boldsymbol{W}(x, y, z) / \partial y \cdot \partial \boldsymbol{P}(x, y, z) /\left.\partial x\right|_{R=R_{0} \Psi\left(\breve{Q}, M_{0}, \Theta, \Phi^{-1}, G^{\prime}, f, \varepsilon^{2}, t\right)} \\
\Delta_{23}^{(i)}(t, \tau)= & \partial \boldsymbol{W}(x, y, z) / \partial y \cdot \partial \boldsymbol{P}(x, y, z) / \partial z- \\
& -\partial \boldsymbol{W}(x, y, z) / \partial z \cdot \partial \boldsymbol{P}(x, y, z) /\left.\partial y\right|_{R=R_{0} \Psi\left(\breve{Q}, M_{0}, \Theta, \Phi^{-1}, G^{\prime}, f, \varepsilon^{2}, t\right)} \\
\Delta_{31}^{(i)}(t, \tau)= & \partial \boldsymbol{W}(x, y, z) / \partial z \cdot \partial \boldsymbol{P}(x, y, z) / \partial x- \\
& -\partial \boldsymbol{W}(x, y, z) / \partial x \cdot \partial \boldsymbol{P}(x, y, z) /\left.\partial z\right|_{R=R_{0} \Psi\left(\breve{Q}, M_{0}, \Theta, \Phi^{-1}, G^{\prime}, f, \varepsilon^{2}, t\right)}
\end{aligned}
$$

where $R=(x, y, z)$ - a vector defined relative to the coordinate system $O_{0} x_{0} y_{0} z_{0}$, connected to the fixed base of the IR.

\section{Conclusions}

As a result, we have the FPC functions $\Delta^{(i)}(t, \tau), \quad(i=1,2, \cdots, n) \quad$ for all links of IR $P_{1}, P_{2}, \cdots, P_{n}$.

The determination of the FPC of the $i$-th link of the IR with the MTE is carried out by checking the condition

$$
\Delta^{(i)}(t, \tau) \neq 0,0<t<T_{0}, \quad \tau=t / \varepsilon
$$

The full FPC of the IR with MTE is determined by checking the conditions (13) for all, i.e. checking the system of conditions

$$
\Delta^{(i)}(t, \tau), \quad 0<t<T_{0}, \quad \tau=t / \varepsilon \quad(i=1,2, \cdots, n) .
$$

Now we can consider the issue of automating the process of selecting effective technological zones for placing PTM components. This problem can be solved by the iteration method used in [10].

The developed mathematical model makes it possible to automate the processes of selecting effective technological zones for placing PTM components with ensuring full FPC not only with MTE, but also with a cumulative system (SS) and thereby all components in the dynamic management of PTM, taking into account production and technological constraints, and can be applied in making production and technological and organizational and structural decisions at the design stage of PTM and PTS as a whole.

In recent years, there has been a tendency in human development to quickly update the old with the new, in particular, the old technology with the new, by using high-level automation methods. The implementation of such an update depends on the operational definition and ensuring their compatibility. 
From this point of view, this paper considers the issues of automation of the selection of effective technological zones for the placement of PTS components with the provision of FPC of their components, in particular PTM.

\section{References}

[1] Mahmood, K., Karaulova, T., Otto, T., \& Shevtshenko E.; Performance Analysis of a Flexible Manufacturing System (FMS). Procedia CIRP, Volume 63, Issue 1, Pp: 424-429, 2017 http://dx.doi.org/10.1016/j.procir.2017.03.123.

[2] Khaleel, A. Modeling and Optimization in a new Machining Production Line by using Manufacturing System Simulation. In Proceedings of the International Conference on Industrial Engineering and Operations Management, Detroit, MI, USA, 23-25 September 2016.

[3] Florescu, A.; Barabas, S. Decision models in designing flexible production systems. In Proceedings of the 4th International Conference on Computing and Solutions in Manufacturing Engineering, Rome, Italy, 3-5 January 2017; Volume 94.

[4] I. R. Belousov, Algoritmi upravleniya robotom manipulyatorom cherez Internet [Algorithms for controlling a robot manipulator via the Internet], Matem. modelirovanie, Volume 14, Issue 8, Pp: 10-15, 2002.

[5] Sahu, A.; Pradhan, S.K.; Quantitative analysis and optimization of production line based on multiple evaluation criteria using discrete event simulation: A review. In Proceedings of the International Conference on Automatic Control and Dynamic Optimization Techniques (ICACDOT), Pune, India, 9-10 September 2016; Pp: 612-617.
[6] Xomchenko V.G., Solomin V.Yu, Yarovoy V. N.; Avtomatizirovannoe reshenie pryamoy zadachi kinematiki manipulyatorov robotov na PEVM[Automated solution of the direct problem of kinematics of robot manipulators on a PC] Metodicheskie ukazaniya $\mathrm{k}$ laboratornoprakticheskim rabotam, Omsk, OmGTU,P: 36, 2007.

[7] Yadav, A.; Jayswal, S.C. Modelling of flexible manufacturing system: A review. Int. J. Prod. Res. 2018, Volume 56, Pp: 2464-2487;

[8] Chimitov P.Ye.; Razrabotka matematicheskoy modeli sborochnix protsessov $\mathrm{s}$ ispolzovaniem metodov raspoznavaniya obrazov[Development of a mathematical model of assembly processes using pattern recognition methods] - autoreferat dissertatsiya na soiskanie uchenoy stepeni kandidata texnicheskix nauk: 05.02.08. - P.E. Chimitov - Irkutsk: NIU GOU VPO «Irkutskiy gosudarstvenniy texnicheskiy universitet», P: 18, 2010.

[9] Erkin, U., Umidjon, M., \& Umida, S. (2021, September). Application of Magnetic Field on Lubricating Cooling Technological Condition in Metal Cutting Process. In International Conference on Reliable Systems Engineering (pp. 100-106). Springer, Cham.

[10] Fota, A.; Flexible Manufacturing Systems Design. Modeling and Simulation; Transilvania University of Brasov Publishing House: Bras, ov, Romania, 2004. (In Romanian).

[11] Umarov, E., Mardonov, U., Abdirakhmonov, K., Eshkulov, A., \& Rakhmatov, B. (2021). Effect of magnetic field on the physical and chemical properties of flowing lubricating cooling liquids used in the manufacturing process. IIUM Engineering Journal, Volume 22, Issue 2, Pp: 327338, 2021.2 doi: https://doi.org/10.31436/iiumej.v22i2.1768.

[12] Rezaie, K., \& Ostadi, B.; (2007). A mathematical model for optimal and phased implementation of flexible manufacturing systems. Applied Mathematics and Computation, Volume 184, Issue $\quad 2, \quad \mathrm{Pp}$ : 729-736. http://dx.doi.org/10.1016/j.amc.2006.05.179. 